\title{
Segurança do Paciente no Contexto da Maternidade: revisão integrativa
}

\author{
Patient Safety in the Maternity Context: integrative review
}

\author{
Ivanete da Silva Santiago Strefling ${ }^{1}$ \\ Marilu Correa Soares ${ }^{2}$ \\ Camila Guadalupe Barcellos ${ }^{3}$ \\ Juliane Portella Ribeiro ${ }^{4}$ \\ Susana Cecagno ${ }^{5}$ \\ Katia da Silva Rocha ${ }^{6}$
}

\begin{abstract}
${ }^{1}$ Enfermeira. Doutoranda do Programa de Pós-Graduação de Enfermagem da Universidade Federal de Pelotas- UFPEL, Pelotas/RS. Docente no curso de Graduação em Enfermagem da Universidade da Região da Campanha - URCAMP, Bagé/RS. Membro do Grupo de Pesquisa e Estudos com Crianças, Adolescentes, Mulheres e Famílias (NUPECAMF). E-mail: ivanete25@gmail.com. Autor correspondente.

${ }^{2}$ Enfermeira Obstetra. Professora Associada da Faculdade de Enfermagem da Universidade Federal de Pelotas/RS. Líder do grupo de Pesquisas e Estudos com Crianças, Adolescentes, Mulheres e Famílias (NUPECAMF). E-mail: enfermeiramarilu@gmail.com.

${ }^{3}$ Enfermeira. Doutora em Enfermagem do Programa de Pós Graduação em Enfermagem da Universidade Federal do Rio Grande - PPGEnf FURG. Chefe do Setor de Gesto do Ensino do Hospital Universitário Miguel Riet Corrêa Jr. E-mail: educação.he@gmail.com.

${ }^{4}$ Enfermeira. Doutora em Enfermagem. Professora adjunta da Faculdade de Enfermagem da Universidade Federal de Pelotas - FEN/UFPEL, Pelotas/RS. E-mail: ju_ribeiro1985@hotmail.com.

${ }^{5}$ Enfermeira. Mestre em Gestão da Qualidade dos Serviços de Saúde - UFRN, Natal/RN. Doutoranda do Programa de Pós-Graduação de Enfermagem da Universidade Federal de Pelotas - UFPEL, Pelotas/RS. Enfermeira Obstetra e Neonatologista da Unidade Materno Infantil HE/UFPEL. E-mail: cecagno@gmail.com.
\end{abstract}

${ }^{6}$ Enfermeira. Mestre em Ciências pelo Programa de Pós-Graduação de Enfermagem da Universidade Federal de Pelotas - UFPEL, Pelotas/RS. Docente na Faculdade de SINOP - FASIPE e Servidora Estadual de Saúde do Mato Grosso. E-mail: katiadasilvarocha@hotmail.com. 


\section{Resumo}

O objetivo é conhecer a produção científica nacional e internacional a respeito da temática segurança do paciente no contexto da maternidade. Trata-se de uma revisão integrativa realizada na Biblioteca Virtual de Saúde utilizando como descritores: segurança do paciente / patient safety / seguridad del paciente e maternidade / maternity / maternidades. Os critérios de inclusão foram: artigos disponíveis na íntegra, online, em português, inglês ou espanhol, publicados no período de 2010 a 2016 que apresentassem informações relevantes. Inicialmente encontrou-se 34 artigos. Após leitura flutuante dos títulos e resumos, selecionou-se 17 estudos para análise textual. Após categorização e análise dos estudos, emergiram as categorias: organização dos serviços de saúde na maternidade, atuação da equipe de saúde frente a qualidade e segurança do atendimento na maternidade e satisfação/insatisfação das mulheres com o atendimento recebido durante a permanência na unidade materna. Foi possível observar nas produções científicas que o cuidado seguro nas unidades materno-infantil depende do bom planejamento e organização da instituição, da comunicação eficiente entre os trabalhadores e da satisfação das mulheres e seus familiares com atenção recebida durante a sua permanência na unidade.

Palavras-chave: Segurança do Paciente; Maternidade; Saúde da Mulher.

\section{Abstract}

The aim is to know the national and international scientific production on the subject of patient safety in the context of maternity. This is an integrative review carried out in the Virtual Health Library using as descriptors: patient safety / patient safety / patient safety and maternity / maternity / maternity. Inclusion criteria were: articles available in full, online, in Portuguese, English or Spanish, published in the period from 2010 to 2016 that presented relevant information. Were initially found 34 articles. After floating reading of the titles and abstracts, we selected 17 studies for textual analysis. After categorizing and analyzing the studies, the following categories emerged: organization of health services in the maternity unit, health team performance in relation to quality and safety of maternity care, and satisfaction/dissatisfaction among women with the care received during the stay in the maternity unit. It was possible to observe in the scientific productions that the safe care in the mother-infant units depends on the good planning and organization of the institution, the efficient communication between the workers and the satisfaction of the women and their families with the attention received during their stay in the unit.

Keywords: Patient Safety; Maternity; Women's Health. 


\section{Introdução}

A segurança do paciente caracteriza-se pela redução do risco de danos desnecessários durante a assistência em saúde ao mínimo aceitável, sendo que um Incidente de Segurança é o evento ou a circunstância que pode resultar ou resultou em dano desnecessário no decorrer do cuidado $^{(1)}$.

O movimento em prol da segurança do paciente teve seu início no final do século XX, após a publicação do relatório To err is Human: building a safer health system, o qual constatou que dentre 33,6 milhões de internações hospitalares de 44.000 a 98.000 pacientes morriam a cada ano nos hospitais dos EUA em virtude dos danos causados durante a prestação de cuidados à saúde ${ }^{(1)}$.

Outro estudo(2) realizado entre 2007 a 2009 em cinco países da América Latina também apontou que 10,5 \% dos pacientes hospitalizados sofrem algum tipo de Evento Adverso, e destes, $58,9 \%$ poderiam ter sido evitados.

A segurança do paciente no contexto da saúde materna e neonatal se reveste de fundamental importância tendo em vista o número de pacientes envolvidos e o potencial de eventos adversos que podem surgir no processo assistencial. Segundo a Organização Mundial de Saúde (OMS) dos 130 milhões de nascimentos que ocorrem todos os anos, cerca de 303 mil resultam na morte da mãe, 2,6 milhões são natimortos e outros 2,7 milhões de recém-nascidos morrem nos primeiros 28 dias após o nascimento ${ }^{(3)}$. Segundo a Dra Marie-Paule Kieny ${ }^{(4)}$, diretora-geral adjunta da OMS "As mulheres e crianças morrem no parto por causas evitáveis, muitas vezes ligados à má qualidade dos cuidados".

No Brasil, acontecem aproximadamente 3 milhões de nascimentos a cada ano, significando quase 6 milhões de pacientes, ou seja, as parturientes e os seus filhos ou filhas ${ }^{(5)}$. Deste total, cerca de $98 \%$ dos partos acontecem em estabelecimentos hospitalares públicos ou privados, sendo os procedimentos obstétricos a terceira causa de internação hospitalar no Sistema Único de Saúde - SUS ${ }^{(4)}$.

Além destes dados, o processo e a natureza do trabalho na assistência materna e neonatal apresenta outras peculiaridades tais como o uso de força de trabalho humano, com várias configurações de equipes, envolvendo médicos, enfermeiros e outros profissionais, que exigem intensiva vigilância para a não ocorrência de erros, além de efetiva comunicação entre diversas disciplinas. Com esses dados, a diversidade de locais de assistência e profissionais envolvidos faz da atenção materna e neonatal uma das áreas prioritárias para ações voltadas à segurança do paciente ${ }^{(6)}$.

Diante do cenário apresentado, governos e organizações internacionais se mobilizaram para apoiar estratégias de prevenção e a mitigação de falhas no cuidado à saúde e divulgar práticas que garantam a segurança do paciente. Nesta intenção, a OMS, apoiada pelos países signatários lançou, em 2004, a Aliança Mundial para Segurança do Paciente. No Brasil foi lançado em abril de 2013 o Programa Nacional de Segurança do Paciente (PNSP) que recomenda implementar protocolos, núcleos de segurança dos pacientes e notificação de eventos adversos no intuito de qualificar o cuidado em todos os estabelecimentos de saúde no território nacional(7).

Especificamente no contexto da atenção obstétrica e neonatal, foi publicado em 2014 o documento Segurança Sanitária em Atenção Materna e Neonatal: segurança e qualidade, voltado para o fortalecimento dos serviços de saúde onde a assistência é prestada, objetivando reduzir os agravos resultantes do próprio processo reprodutivo e minimizar os danos relacionados ao processo assistencial, além de contribuir para uma assistência que tenha como focos principais a segurança e a humanização( ${ }^{(8)}$.

Considerando que a melhoria da qualidade e segurança na assistência materna e neonatal devem incluir todas as estratégias voltadas à assistência aos pacientes em geral assim como estratégias específicas para esse grupo e a importância do desenvolvimento de pesquisas baseadas em evidências científicas com melhores práticas voltadas à segurança do paciente, objetivou-se neste estudo: conhecer a produção científica nacional e internacional a respeito da temática segurança do paciente no contexto da maternidade.

\section{Método}


Trata-se de uma revisão integrativa de literatura, a qual se caracteriza por ser um método que prevê a análise de pesquisas, subsidiando a tomada de decisão e permitindo compreensão e o conhecimento de um determinado assunto. Permite ainda o reconhecimento de lacunas no conhecimento que necessitam ser preenchidas por meio de novas pesquisas ${ }^{(9)}$.

Este tipo de pesquisa consiste na organização, catalogação e síntese dos resultados apresentados nos materiais selecionados para análise, facilitando a interpretação. Uma revisão integrativa constituiu-se na busca de dados em fontes secundárias, porém, adotando o mesmo rigor e clareza de um estudo primário. Para tanto, foram adotadas as seguintes etapas: definição da questão de pesquisa; busca nas bases de dados; categorização dos estudos; avaliação dos estudos incluídos na revisão; interpretação dos resultados; síntese do conhecimento produzido ${ }^{(10)}$.

A questão que norteou esta pesquisa foi: qual a produção científica, nacional e internacional, disponível na Biblioteca Virtual em Saúde (BVS) a respeito da segurança do paciente no contexto da maternidade?

Das publicações localizadas na BVS os artigos encontraram-se hospedados nas bases de dados Literatura Latino-Americana e do Caribe em Ciências da Saúde (LILACS), Base de Dados de
Enfermagem (BDENF) e National Library of Medicine National Institutes of Health (MEDLINE).

Os critérios de inclusão foram: artigos disponíveis na íntegra, online, em português, inglês ou espanhol, publicados no período de 2010 a 2016 e que apresentassem informações relevantes ao tema de pesquisa. Utilizaram-se como descritores: segurança do paciente / patient safety / seguridad del paciente e maternidades / maternity / maternidades.

Após leitura detalhada de todos os artigos, foi elaborado um quadro contendo: número para identificação do artigo, título do artigo, autores, periódico/base de dados e ano de publicação. Para a avaliação dos estudos, utilizou-se a Análise Textual Qualitativa, a qual se desenvolve mediante um processo de fragmentação do material lido ${ }^{(11)}$.

\section{Resultados e Discussão}

Foram encontrados 34 artigos na BVS, de acordo com os critérios de inclusão, sendo que dois eram repetidos, permanecendo 32 para a préanálise. A seleção da amostra foi realizada por meio de leitura flutuante dos títulos e resumos seguida da leitura dos artigos na íntegra. Após esta etapa, selecionou-se 17 artigos que estavam de acordo com a temática em questão. Destes, 12 encontravam-se hospedados na base de dados da MEDLINE, 3 no LILACS e 2 na BDENF.

Quadro 1. Síntese dos artigos. Pelotas, RS, Brasil, 2017.

\begin{tabular}{|c|c|c|c|c|}
\hline $\mathrm{N}^{\circ}$ & TITULO & AUTORES & PERIÓDICO/ BASE & ANO \\
\hline \multirow[t]{2}{*}{1} & $\begin{array}{l}\text { Reducing the length of postnatal hospital stay: } \\
\text { implications for cost and quality of care }\end{array}$ & $\begin{array}{l}\text { Bowers, J; } \\
\text { Cheyne, } \mathrm{H} \text {. }\end{array}$ & $\begin{array}{l}\text { BMC Health Serv } \\
\text { Res / MEDLINE }\end{array}$ & 2016 \\
\hline & $\begin{array}{l}\text { The health team and the safety of the mother- } \\
\text { baby binomial during labor and birth }\end{array}$ & $\begin{array}{l}\text { Dornfeld, D; } \\
\text { Pedro, ENR. }\end{array}$ & $\begin{array}{l}\text { Invest Educ } \\
\text { Enferm / } \\
\text { LILACS/BEDEnF }\end{array}$ & 2015 \\
\hline 3 & $\begin{array}{l}\text { Lessons learned from the introduction of an } \\
\text { electronic safety net to enhance test result } \\
\text { management in an Australian mothers' hospital }\end{array}$ & $\begin{array}{l}\text { Georgiou, A; Lymer, } \\
\text { S; Forster, } \\
\text { M;Strachan, M; } \\
\text { Graham, S; Hirst, } \\
\text { G;Callen, } \\
\text { Westbrook, Jl. }\end{array}$ & $\begin{array}{l}\text { J Am Med Inform } \\
\text { Assoc / MEDLINE }\end{array}$ & 2014 \\
\hline 4 & $\begin{array}{l}424 \text { Membres de la commission des usagers du } \\
\text { Reseau "Securite NaissanceNaitre ensemble 1/4 } \\
\text { des Pays-de-la-Loire }\end{array}$ & $\begin{array}{l}\text { Branger, B; Le Coz, } \\
\text { F; Gillard, P; Merot, } \\
\text { E; Winer, N. }\end{array}$ & $\begin{array}{l}\text { J Gynecol Obstet } \\
\text { Biol Reprod / } \\
\text { MEDLINE }\end{array}$ & 2014 \\
\hline
\end{tabular}




\begin{tabular}{|c|c|c|c|c|}
\hline 5 & $\begin{array}{l}\text { Perinatal staff perceptions of safety and quality } \\
\text { in their service }\end{array}$ & $\begin{array}{l}\text { Sinni, SV; Wallace, } \\
\text { EM; Cross, WM. }\end{array}$ & $\begin{array}{l}\text { BMC Health Serv } \\
\text { Res / MEDLINE }\end{array}$ & 2014 \\
\hline 6 & $\begin{array}{l}\text { Avaliação da adesão ao checklist de cirurgia } \\
\text { segura da OMS em cirurgias urológicas e } \\
\text { ginecológicas, em dois hospitais de ensino de } \\
\text { Natal, Rio Grande do Norte, Brasil }\end{array}$ & $\begin{array}{l}\text { Freitas, MR; Monte, } \\
\text { LC; AAG; Lopes, } \\
\text { BNA; Fernandes, } \\
\text { FC; Gama, ZAS. }\end{array}$ & $\begin{array}{l}\text { Cad Saúde Pública } \\
\text { / LILACS }\end{array}$ & 2014 \\
\hline 7 & $\begin{array}{l}\text { Room for improvement: noise on a maternity } \\
\text { ward }\end{array}$ & $\begin{array}{l}\text { Adatia,S; Law, } \\
\text { S; Haggerty, J. }\end{array}$ & $\begin{array}{l}\text { BMC Health Serv } \\
\text { Res / MEDLINE }\end{array}$ & 2014 \\
\hline 8 & $\begin{array}{l}\text { Changes in care associated with the } \\
\text { introduction of a postpartum hemorrhage } \\
\text { patient safety program }\end{array}$ & $\begin{array}{l}\text { Lappen, JR; } \\
\text { Seidman, D; } \\
\text { Burke, C; } \\
\text { Goetz, K; } \\
\text { Grobman, WA. }\end{array}$ & $\begin{array}{l}\text { Am J Perinatol; } \\
\text { MEDLINE }\end{array}$ & 2013 \\
\hline 9 & $\begin{array}{l}\text { Nurses' perspectives on the intersection of } \\
\text { safety and informed decision making in } \\
\text { maternitycare }\end{array}$ & $\begin{array}{l}\text { Jacobson, } \mathrm{CH} \text {; } \\
\text { Zlatnik, MG; } \\
\text { Kennedy, H P; } \\
\text { Lyndon, A. }\end{array}$ & $\begin{array}{l}\text { J Obstet } \text { Gynecol } \\
\text { Neonatal } \\
\text { MEDLINE; }\end{array}$ & 2013 \\
\hline 10 & $\begin{array}{l}\text { Managing information and knowledge within } \\
\text { maternity services: Privacy and consent issues }\end{array}$ & $\begin{array}{l}\text { Baskaran, V; } \\
\text { Davis, K; } \\
\text { Bali, RK; } \\
\text { Naguib, RNG; } \\
\text { Wickramasinghe N. }\end{array}$ & $\begin{array}{l}\text { Inform Health Soc } \\
\text { Care/ MEDLINE }\end{array}$ & 2013 \\
\hline 11 & $\begin{array}{l}\text { Women's safety alerts in maternity care: is } \\
\text { speaking up enough? }\end{array}$ & $\begin{array}{l}\text { Rance, S; } \\
\text { McCourt, C; } \\
\text { Rayment, } \\
\text { Mackintosh, J; } \\
\text { Carter, W; Watson, K; } \\
\text { Sandall, J. }\end{array}$ & $\begin{array}{l}\text { BMJ Qual Saf; } \\
\text { MEDLINE }\end{array}$ & 2013 \\
\hline 12 & $\begin{array}{l}\text { Percepção da mulher sobre o processo de } \\
\text { nascimento acompanhado }\end{array}$ & $\begin{array}{l}\text { Palinski, JR; } \\
\text { Souza, SRRK; Silveira, } \\
\text { JTP; Salim, NR; } \\
\text { Gualda, DMR. }\end{array}$ & $\begin{array}{l}\text { Online } \quad \text { Brazilian } \\
\text { Journal of } \\
\text { Nursing/ BDENF }\end{array}$ & 2012 \\
\hline 13 & $\begin{array}{l}\text { Management processes as a tool for quality in a } \\
\text { public hospital-maternity in the city of São Paulo }\end{array}$ & Demarchi, TM. & $\begin{array}{l}\text { Rev. adm. Saúde/ } \\
\text { LILACS }\end{array}$ & 2012 \\
\hline 14 & $\begin{array}{l}\text { Patients' perceptions of safety and quality of } \\
\text { maternity clinical handover }\end{array}$ & $\begin{array}{l}\text { Chin, GSM; Warren, } \\
\text { N; Kornman, } \\
\text { L; Cameron, P. }\end{array}$ & $\begin{array}{l}\text { BMC Pregnancy } \\
\text { Childbirth; } \\
\text { MEDLINE }\end{array}$ & 2011 \\
\hline 15 & $\begin{array}{l}\text { Midwife-led care unit for 'low risk' pregnant } \\
\text { women in a Japanese hospital }\end{array}$ & $\begin{array}{l}\text { Suzuki, S; Hiraizumi, } \\
\text { Y; Satomi, M; Miyake, } \\
\text { H. }\end{array}$ & $\begin{array}{ll}\text { J Matern } & \text { Fetal } \\
\text { Neonatal } & \text { Med; } \\
\text { MEDLINE } & \end{array}$ & 2011 \\
\hline 16 & $\begin{array}{l}\text { A comunicação como fator de segurança e } \\
\text { proteção ao parto }\end{array}$ & $\begin{array}{l}\text { Dornfeld, D; Pedro, } \\
\text { ENR. }\end{array}$ & $\begin{array}{l}\text { Rev. } \quad \text { Eletrônica } \\
\text { enferm; LILACS }\end{array}$ & 2011 \\
\hline 17 & $\begin{array}{l}\text { Supporting patient safety: examining } \\
\text { communication within delivery suite teams } \\
\text { through contrasting approaches to research } \\
\text { observation }\end{array}$ & $\begin{array}{l}\text { Berridge, } \\
\text { EJ; Mackintosh, } \\
\text { NJ; Freeth, DSM }\end{array}$ & MEDLINE & 2010 \\
\hline
\end{tabular}

Fonte: dados da pesquisa. 
Dentre os estudos analisados, $82,32 \%$ foram publicados em base de dados internacional, enquanto que apenas $17.68 \%$ foram nacionais. $\mathrm{Da}$ produção total, cinco artigos foram realizados em serviços de saúde brasileiros, 12 são oriundos de instituições estrangeiras e dois são reflexões teóricas. Frente a esta observação, nota-se que, no Brasil, a produção científica na área de segurança do paciente voltada para a área materna e neonatal ainda é incipiente.

Quanto a abordagem metodológica utilizada 8 artigos enfocaram o método qualitativo, 6 utilizaram abordagem quantitativa, 1 estudo quali-quantitativo e um de pesquisa documental.

Em relação ao conteúdo, após a categorização e análise textual dos artigos, emergiram três categorias. Uma refere-se à organização dos serviços de saúde na maternidade, outra faz referencia a atuação da equipe de saúde frente à qualidade e segurança do atendimento na maternidade e a terceira categoria evidenciou assuntos relacionados à satisfação/insatisfação das mulheres com o atendimento recebido durante a permanência na unidade materna.

\section{maternidade}

Organização dos serviços de saúde na

Nesta categoria foram reunidos os estudos que enfocam a importância da estrutura física, dos recursos humanos e do processo de trabalho e assistencial na organização do serviços de saúde na maternidade em prol da segurança do paciente.

Um estudo ${ }^{(12)}$ que avaliou os fatores contribuintes para o elevado índice de morte materna na África, pesquisadores apontou que o fator de maior índice de insegurança no cuidado as mulheres e recém-nascidos estava relacionado à falta de organização dos serviços de saúde para implementar políticas, protocolos ou diretrizes.

No Brasil, sobretudo a partir do ano 2000, se intensificam ações com o intuito de reduzir as altas taxas de morbimortalidade materna e perinatal com o Programa Humanização do Prénatal e Nascimento - PHPN ${ }^{(13)}$, com a Rede Cegonha ${ }^{(5)}$ em 2011 e em 2014 a ANVISA lançou o documento Serviços de Atenção Materna e Neonatal: segurança e qualidade ${ }^{(8)}$.
As duas primeiras estratégias representam um conjunto de iniciativas que envolvem mudanças no processo de cuidado à gravidez, ao parto e nascimento, articulando os pontos de atenção em rede à regulação obstétrica. Sua operacionalização requer qualificação técnica das equipes da atenção básica e das maternidades, melhoria da ambiência dos serviços de saúde e ampliação da oferta de serviço e do número de profissionais ${ }^{(14)}$

A última vem ao encontro das necessidades dos gestores, fiscais de vigilância sanitária e profissionais envolvidos na assistência, servindo de base e orientação para a construção e reforma de unidades de atenção materna e neonatal além de guia para a organização e estruturação dos serviços. Também serve de apoio e referência para a construção de sistemas de segurança do paciente nessas unidades com vistas à redução de erros e danos inerentes ao processo assistencial ${ }^{(8)}$.

Neste contexto, autores ${ }^{(15-16)}$ assinalaram que a qualidade do cuidado nos serviços de saúde depende de uma articulação complexa do processo de trabalho, da equipe multidisciplinar, das condições de infraestrutura e do sistema de saúde tendo a gestão um papel importante nesta articulação. Outro estudo ${ }^{(17)}$ apontou a necessidade de redesenhar os processos assistenciais para a racionalização no trabalho, a satisfação dos envolvidos, a inovação e, consequentemente, a melhoria da qualidade da assistência e segurança das puérperas e recémnascidos.

Outras medidas identificadas foram a implementação de registros eletrônicos e governanças de informações para manter a privacidade, a confidencialidade e segurança ${ }^{(18-19)}$. Segundo estudo realizado numa maternidade da Inglaterra, foi identificado violações no compartilhamento de informações pessoais sem o consentimento das pacientes ${ }^{(20)}$.

Foi avaliada a implementação de medidas de segurança e melhoria do cuidado as parturientes e RNs como a adesão ao checklist em cirurgias urológicas e ginecológicas de dois hospitais de ensino em Natal, Rio Grande do Norte/Brasil. Do total de cirurgias, somente $61 \%$ 
tinham checklist e $4 \%$ delas estavam totalmente preenchidos $^{(21)}$.

Os outros estudos que enfocaram a questão do conforto e do silêncio para a adequada recuperação da parturiente e favorecimento da formação de vínculo com o neonato observaram que as novas mães enfrentam constantes interrupções ao longo do dia, incluindo visitas por uma variedade de profissionais de saúde, funcionários do hospital, estudantes, familiares e amigos. Além disso, a presença constante de ruídos resultantes do manejo de equipamentos médicos, conversas de corredor, anúncios de intercomunicador, construção, abertura e fechamento de portas, equipamentos de limpeza e carrinhos de alimentação ${ }^{(22-23)}$.

Isso significa rupturas em atividades de aprendizagem importantes, como a amamentação, que é fundamental para estabelecer vínculo da mãe com seu bebê e o fortalecimento do aleitamento materno exclusivo nos primeiros dias do parto. Um ambiente altamente perturbador também pode levar à privação aguda do sono, aumentando o risco de distúrbios de saúde mental pós-parto e resultando em disfunções cardiovasculares $^{(24)}$.

Segundo dispõe o PHPN, o local destinado à atenção a mulher no trabalho de parto e parto é fundamental para o sucesso deste processo. Portanto, proporcionar um ambiente acolhedor, organizando o local, tornando-o agradável e seguro para a mulher, tende a diminuir a ansiedade, a insegurança, além de facilitar a presença do acompanhante e a formação de vínculo.

\section{Dificuldades e facilidades da equipe de saúde para prestar cuidado seguro do na maternidade}

Identificou-se nesta categoria aspectos relacionados as dificuldades e as facilidades para a equipe de saúde oferecer um cuidado seguro e de qualidade às mulheres e recém-nascidos durante sua permanência na maternidade.

A comunicação se destacou como um dos fatores fundamentais para a melhoria da segurança das parturientes e dos recém-nascidos. A complexidade dos padrões de comunicação e as múltiplas influências nos modelos e normas institucionais acarretam tensões interprofissionais, pressões da carga de trabalho, entraves com implicações para a segurança(25).

Autores $^{(26-27)}$ observaram 10 cenas de parto e identificaram comunicação iatrogênica, apesar de perceberam apoio empático e incentivo à presença do acompanhante. Destacaram ainda a relevância que a equipe de saúde tem na segurança e proteção da mulher e do RN e, sobretudo da importância da enfermeira como agente de mudança para um modelo de atenção à saúde focado nas necessidades da mulher-RNfamília.

Em relação ao Contato Pele a Pele (CPP) entre mãe e bebê e a disposição de um ambiente propício para a recepção do recém-nascido,

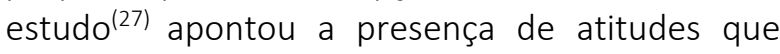
demonstraram a conscientização dos profissionais de saúde, com destaque para a enfermagem, sobre os benefícios dessas práticas, no entanto, ainda há necessidade de esforços para que estas ações se configurem em circunstâncias seguras de cuidado.

Ao buscar entender melhor como os desafios de comunicação interdisciplinar podem afetar a segurança das puérperas e dos recémnascidos, estudo realizado nos Estados Unidos evidenciou que as ações dos enfermeiros para evitar danos as pacientes abrangem o convencimento de acordo, o gerenciamento de informações e a capacitação de mães e médicos ${ }^{(28)}$.

Essas ações foram executadas em um contexto complexo e hierárquico caracterizado por padrões e prática de relacionamentos variados. As prioridades dos enfermeiros e as metas de segurança dos pacientes eram, por vezes, desalinhadas com as dos médicos, resultando em comunicação potencialmente insegura(28).

Para autores(29), o obstetra tem grande oportunidade de ser uma influência, positiva e ou negativa sobre o cuidado seguro no período perinatal. No entanto, a dinâmica da equipe, a compreensão, o respeito mútuo e a confiança consistem em questões fundamentais para a potencial melhoria de serviço futuro. Além da comunicação iatrogênica, processos de trabalho robustos de cuidado podem dificultar a assistência segura e de qualidade às mulheres e aos recémnascidos nas unidades maternas. 
O PNHN estabelece que os partos de baixo risco podem ser acompanhados por enfermeiras obstetras ancorados em evidências científicas que discutem o modelo assistencial e são favoráveis à inclusão da enfermeira obstétrica no acompanhamento da gestação, trabalho de parto e parto de baixo risco ${ }^{(13)}$.

Neste contexto, ao comparar os resultados obstétricos de 1031 gestantes de baixo risco, que foram admitidas na maternidade, assistida por enfermeira obstétrica com relação aos cuidados compartilhados com a equipe médica obstétrica não houve nenhuma evidência indicando que os cuidados da enfermeira obstétrica, não seja seguro para a atenção das gestantes de baixo risco( ${ }^{(30)}$.

No Brasil, o enfermeiro obstetra tem respaldo legal para realizar todas as atividades de enfermagem na área de obstetrícia, cabendo-Ihe privativamente executar parto sem distócia desde a legalização do exercício profissional, conforme consta na Resolução no 0477/2015 que dispõe sobre a atuação de enfermeiros na assistência às gestantes, parturientes e puérperas ${ }^{(31)}$.

\section{Satisfação/insatisfação das mulheres com o atendimento recebido durante sua permanência na unidade materna}

Nesta categoria foram

congregados estudos que fizeram referencia a perspectiva das mulheres com o atendimento recebido. Apontam-se tanto os fatores de satisfação quanto os aspectos de insatisfação das mulheres com o cuidado recebido durante sua permanência da maternidade.

Estudo realizado na França( ${ }^{(32)}$ observou que das mulheres que tiveram parto cesariana e as que tiveram partos normais, 92,5\% mostraram-se satisfeitas com 0 atendimento recebido na maternidade. Dentre os fatores de satisfação destacaram as orientações sobre os cuidados com o bêbe e a amamentação ${ }^{(32)}$. Um estudo ${ }^{(33)}$ ressaltou, ainda, a presença do acompanhante como fator positivo para as mulheres porque proporciona segurança, tranquilidade, apoio físico, gratidão e emoção.

A presença do acompanhante de escolha da parturiente durante o trabalho de parto, parto e pós-parto imediato, no âmbito do SUS é um direito garantido pela Lei n. $011.108^{(34)}$.
Contudo, dentre os fatores de insatisfação, mulheres relataram sentimentos negativos como solidão e medo devido à ausência do acompanhante em algumas das etapas do cuidado. Algumas manifestaram, ainda, sentimento de pressão no momento do parto quando o acompanhante pede para fazer força e ela se sente constrangida(33).

Em um estudo ${ }^{(35)}$, mulheres hesitam em falar sobre preocupações e angustias por pensar que a equipe pode considerar irrelevante e não as ouvir, assim como a demorar para dar feedback. Outro fator de insatisfação descrito se refere à falta de escolha da maternidade e a existência de intervenções desnecessária como a episiotomia ${ }^{(32)}$.

Ao descrever as boas práticas e as intervenções obstétricas empregadas na assistência a mulheres de risco obstétrico habitual,

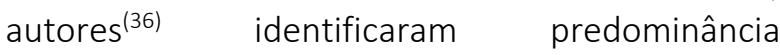
significativa de intervenções desnecessárias em relação às boas práticas obstétricas. Entre as mulheres avaliadas, 92\% delas foram postas em posição de litotomia, em $37 \%$ foi aplicada a manobra de Kristeller, em 56\% foi realizado episiotomia, 40\% utilizaram ocitocina e foram submetidas à aminiotomia e $30 \%$ à analgesia raqui/epidural. Por outro lado, somente $45 \%$ das mulheres deambularam durante o trabalho de parto e foram monitoradas por meio do partograma.

A despeito da importância da presença do acompanhante em todo o processo gestacional, prática que deve ser estimulada durante todas as atividades, tanto de consultas pré-natais como em grupos de educação em saúde, ainda se encontra limitada por diversos fatores.

Um destes fatores está relacionado às questões de organização e ambiente nas instituições. Em um dos estudos analisados(33), a maternidade não dispunha de quartos privativos para que a mulher pudesse vivenciar o momento da parturição com privacidade.

Outro artigo ressaltou a importância da autonomia da parturiente como mecanismos de proteção que aumenta a qualidade e segurança de cuidado. A autonomia pode ser adquirida por meio do empoderamento da mulher no decorrer da gestação. Na Inglaterra, mulheres assistidas no pós-parto referiram que por terem sido 
informadas sobre seus direitos, por terem desenvolvido um plano de parto com a cooperação da equipe de saúde, sentiram-se mais seguras e acolhidas e mais envolvidas na tomada de decisões ${ }^{(37)}$.

Ao fazer um paralelo entre o modelo de atenção obstétrica e neonatal predominante no Brasil, em relação aos atributos da qualidade e segurança na assistência à saúde preconizado pela OMS e as metas internacionais de segurança do paciente propostas pela Aliança Mundial pela Segurança do Paciente, é possível observar que embora haja uma preocupação crescente em fomentar iniciativas para a melhoria na rede de atenção materna e neonatal, indicadores de resultados da assistência obstétrica demonstram a gravidade dos danos sofridos pelas mulheres assistidas, bem como à baixa qualidade da assistência ${ }^{(3-7-38)}$.

Segundo tese realizada em $2015^{(39)}$, o modelo de atenção obstétrica predominante no país, ainda é fortemente intervencionista, focado na hegemonia médica, sem transposição das evidências científicas para a prática. Os serviços de saúde não promovem uma assistência respeitosa, pois desconsideram as preferências, necessidades e valores individuais das mulheres assistidas, predominando decisões, muitas vezes arbitrárias e não justificadas, dos profissionais que realizam o cuidado durante o processo de parturição.

Dados apontam que as mulheres ainda são submetidas a uma peregrinação quando buscam os serviços de saúde para serem assistidas durante o trabalho de parto e parto, o que aumentam significativamente o risco de danos graves, como a morte materna e do neonato. Também persiste a utilização indiscriminada de tecnologias e intervenções desnecessárias, promovendo o desperdício no uso de equipamentos, suprimentos, ideias e energia ${ }^{(36)}$.

Diante das evidências científicas expostas nesta revisão, corrobora-se com Vicent ${ }^{(40)}$ que a segurança no processo assistencial precisa ser enfocada na perspectiva da evitabilidade, prevenção e melhoria de resultados adversos ou lesões resultantes do cuidado em saúde.

Destarte, fragilidades como as apontadas, nesta revisão integrativa, podem comprometer a segurança e a qualidade do cuidado necessário às mulheres em processo de parturição e ao seu filho, justificando a necessidade de um olhar sistêmico para todos os aspectos que envolvem o processo assistencial desta população específica.

\section{Conclusão}

O resultado desta revisão integrativa, que buscou conhecer a produção científica relacionada à segurança do paciente no contexto da maternidade, mostrou a crescente preocupação com a questão da qualidade e segurança materna e neonatal confirmado pelo aumento significativo na produção científica nacional e internacional em relação ao referido tema nos últimos anos.

Foi possível perceber que os estudos apontaram fatores que envolvem o sistema institucional como um todo. Identificou-se características relacionadas a organização do serviço e da infraestrutura, fatores que envolvem a gestão e o processo de trabalho de equipes interdisciplinares, comunicação interpessoal, adoção de medidas de conforto e segurança as mulheres e neonatos e a satisfação/insatisfação das mulheres e ou familiares com o contexto de cuidado e assistência recebida, assim como o uso indiscriminado de intervenções desnecessárias.

Diante desta realidade, evidencia-se a importância de aprofundar investigações que tenham uma perspectiva sistêmica sobre todas as barreiras presentes nos serviços de saúde que podem resultar em situações de risco a qualidade e segurança do cuidado prestado em prol da parturiente e do recém-nascido.

\section{Referências}

1. World Health Organization (WHO). Global Priorities for patient safety research [Internet]. 2009 [acesso em 08 out 2017]. Disponível em: http://www.who.int/patientsafety/research/priori ties.

2. Ministrio de Sanidad y Politica Social IBERO (ES). Estudio IBEAS: prevalencia de efectos adversos en hospitales de Latinoamérica [Internet]. 2010 [acesso em 10 out 2017]; 182pg. Disponível

em: 
http://www.msc.es/organizacion/sns/planCalidad SNS/docs/INFORMEIBEAS.pdf.

3. World Health Organization (WHO). Women and health: today's evidence tomorrow's. 2009b.

4. Instituto Brasileiro de Segurança do Paciente (BR). OMS cria checklist de parto seguro que visa segurança do paciente [Internet]. 2016 [acesso em 23 out 2017]. Disponível em: https://www.segurancadopaciente.com.br/no ticia/oms-cria-checklist-de-parto-seguro-quevisa-seguranca-do-paciente/.

5. Ministério da Saúde (BR). Portaria n.o 1.459, de 24 de junho de 2011. Institui no âmbito do Sistema Único de Saúde - SUS - a Rede Cegonha. Diário Oficial da União, Brasília [Internet]. 2011 [acesso em 15 jun 2017]. Disponível em: <http://bvsms.gov.br/bvs/ saudelegis/gm/201 1/prt145924062011.html>.

6. Scarrow PK. Patient safety in obstetrics and beyond. J. Healthcare Qual. 2009; 31(5).

7. Ministério da Saúde (BR). Documento de Referência para o Programa Nacional de Segurança do Paciente. Fundação Oswaldo Cruz; Agência Nacional de Vigilância Sanitária. - Brasília; 2014. 40 p.

8. Agência Nacional de Vigilância Sanitária (BR). Serviços de atenção materna e neonatal: segurança e qualidade. Brasília; 2014. 103 p.

9. Mendes KS, Silveira RCCP, Galvao, CM. Revisão integrativa: método de pesquisa para a incorporação de evidências na saúde e na enfermagem. Texto Context Enferm. 2008; 17(4):758-764.
10. Ganong LH. Integrative review of nursing research. Res Nursing Health, 1987; 10(1):1-11.

11. Moraes R. Mergulhos discursivos: análise textual qualitativa entendida como processo integrado de aprender, comunicar e interferir em discursos In: Galiazzi MC, Freitas JV, organizadores. Metodologias emergentes de pesquisa em educação ambiental. Ijuí: Ed. Unijuí; 2005.

12. Madzimbamuto FD, Ray SC, Mogobe KD, Ramogola-Masire D, Phillips R, Haverkamp M, Mokotedi M, Motana M. A rootcause analysis of maternal deaths in Botswana: towards developing a culture of patient safety and quality improvement. BMC Pregnancy Childbirth [Internet]. 2014 [acesso em 20 out 2017]; 16(14):231. Disponível em: https://doi.org/10.1186/1471-2393-14-231.

13. Ministério da Saúde (BR). Portaria/GM n.o 569, de 1 을 de junho de 2000. Institui o Programa de Humanização no Pré-Natal e Nascimento, no âmbito do Sistema Único de Saúde [Internet]. Brasília; 2000. [acesso em 19 de out 2017]. Disponível em: http://dtr2001.saude.gov.br/sas/PORTARIAS/POR T2000/GM/GM-569.htm.

14. Martinelli KG, Neto ETS, Gama SGN, Oliveira AE. Adequação do processo da assistência pré-natal segundo os critérios do Programa de Humanização do Pré-natal e Nascimento (PHPN) e Rede Cegonha. Rev Bras Ginecol Obstet. 2014; 36(2):56-64.

15. Magluta C, Noronha MF, Gomes MAM, Aquino LA, Alves CA, Silva RS. Estrutura de maternidades do Sistema Único de Saúde do Rio de Janeiro: desafio à qualidade do cuidado à saúde. Rev Bras Saúde Mater Infant [Internet]. 2009 [acesso em 21 out 2017]; 9(3):319-329. Disponível 
em: http://dx.doi.org/10.1590/S1519-

38292009000300011.

16. Bowers J, Cheyne H. Reducing the length of postnatal hospital stay: implications for cost and quality of care. BMC Health Serv Res [Internet]. 2016 [acesso em 13 out 2017]; 16(16). Disponível em: https://doi.org/10.1186/ s12913-015-1214-4.

17. Demarchi TM. Management processes as a tool for quality in a public hospital-maternity in the city of São Paulo. Rev adm. Saúde. 2012; 14(54):37-43.

18. Clemens NA. Privacy, consent, and the electronic mental health record: the person vs. the System. J Psychiatr Pract. 2012; 18(1):46-50.

19. Georgiou A, Lymer S, Forster M, Strachan M, Graham S, Hirst G, Callen J, Westbrook Jl. Lessons learned from the introduction of an electronic safety net to enhance test result management in an Australian mothers' hospital. J Am Med Inform Assoc. 2014; 21(6):1104-8.

20. Baskaran V, Davis K, Bali RK, Naguib RNG, Wickramasinghe N. Managing information and knowledge within maternity services: privacy and consent issues. Inform Health Soc Care. 2013; 38(3):196-210.

21. Freitas MR, Monte LC, Antunes AG, Lopes BNA, Fernandes FC, Monte LC, Gama ZAS. Avaliação da adesão ao checklist de cirurgia segura da OMS em cirurgias urológicas e ginecológicas, em dois hospitais de ensino de Natal, Rio Grande do Norte, Brasil. Cad Saúde Pública [Internet]. 2014 [acesso em 18 out 2017]; 30(1):137-148. Disponível em: http:// dx.doi.org/10.1590/0102-311X00184612.

22. Morrison B, Ludington-Hoe S. Interruptions to breastfeeding dyads in an DRP unit.
MCN Am J Matern Criança Nurs [Internet]. 2012 [acesso em 24 out 2017]; 37(1):36-41. Disponível em:

https://www.ncbi.nIm.nih.gov/pubmed/22157339

23. Adatia S, Law S, Haggerty J. Room for improvement: noise on a maternity ward. BMC Health Serv Res. 2014; 14:604.

24. Sauvet F, Leftheriotis G, GomezMerino D, Langrume C, Drogou C, Van Beers P, Bourrilhon C, Florence G, Chennaoui M. Effect of acute sleep deprivation on vascular function in healthy subjects. J Appl Physiol. 2010; 108(1):68-75.

25. Berridge E, Mackintosh NJ, Freeth DSM. Supporting patient safety: examining communication within delivery suite teams through contrasting approaches to research observation. Midwifery. 2010; 26(5):512-9.

26. Dornfeld D, Pedro ENR. A comunicação como fator de segurança e proteção ao parto. Rev Eletr Enferm. 2011; 13(2).

27. Dornfeld D, Pedro ENR. The health team and the safety of the mother-baby binomial during labor and birth. Invest Educ Enferm. 2015; 33(1):44-52.

28. Jacobson $\mathrm{CH}$, Zlatnik MG, Kennedy HP, Lyndon A. Nurses' perspectives on the intersection of safety and informed decision making in maternity care. J Obstet Gynecol Neonatal Nurs. 2013; 42(5):577-87.

29. Suzanne VS, Wallace EM, Cross WM. Perinatal staff perceptions of safety and quality in their service. BMC Health Serv Res. 2014; 14:591.

30. Suzuki S, Hiraizumi $Y$, Satomi $M$, Miyake H. Midwife-led care unit for 'low risk' pregnant women in a Japanese hospital. J Matern Fetal Neonatal Med. 2011; 24(8):1046-50. 
31. Conselho Federal de Enfermagem - COFEN. Resolução n.o 0477/2015 que dispõe sobre a atuação de enfermeiros na assistência às gestantes, parturientes e puérperas. 2015.

32. Branger $A B$, Le CFB, Gillard $C P$, Merot DE, Winer N. Satisfaction de 424 usagers pendant la grossesse et à l'accouchement dans le Réseau de santé en périnatalité - Sécurité Naissance - des Pays-de-la-Loire. Journal of Obstetrics Ginecologia e Reprodutiva Biologia. 2014; 43(5):361-370.

33. Palinski JR et al. Percepção da mulher sobre $\mathrm{O}$ processo de nascimento acompanhado. Online Brazilian Journal of Nursing [Internet]. 2012 [acesso em 22 nov 2016]; 11(2):274-88. Disponível em: http://www.objnursing.uff.br/index.php/nursing/a rticle/view/3603.

34. Ministério da Saúde (BR). Lei n.o 11.108, de 7 de abril de 2005. Lei do acompanhante; 2005.

35. Rance S, Mccourt C, Rayment J, Mackintosh N, Carter W, Watson K, Sandall J. Women's safety alerts in maternity care: is speaking up enough? BMJ Qual Saf. 2013; 22(4):348-55.

36. Leal $M C$ et al . Intervenções obstétricas durante o trabalho de parto e parto em mulheres brasileiras de risco habitual. Cad. Saúde Pública. 2014; 30(Sup.1):S17-S32.

37. Chin GSM, Warren N, Kornman L, Cameron P. Patients' perceptions of safety and quality of maternity clinical handover. BMC Pregnancy Childbirth. 2011; 11(58).

38. Lansky $\mathrm{S}$ et al. Pesquisa nascer no Brasil: perfil da mortalidade neonatal e avaliação da assistência à gestante e ao recém-nascido. Cad Saúde Pública. 2014; 30(Supl.1):192-207.

39. Melo CR. Adaptação transcultural do Maternity Safety Thermometer para o português do Brasil. [Tese] Programa de Pós-
Graduação em Enfermagem, Universidade Federal de Santa Catarina, Florianópolis, 2015. 291f.

40. Vincent C. The essentials of patient safety. London: Imperial College; 2011. 